\title{
New techniques to determine structural transformations of active catalysts
}

Jonathan Hanson ${ }^{1}$, Sanjaya D. Senanayake ${ }^{1}$, David Salazar ${ }^{1}$, milinda Abeykoon ${ }^{2}$, Goknur Tutuncu ${ }^{2}$, Andrey Yakovenko $^{3}$ ${ }^{1}$ Chemistry Department, Brookhaven National Laboratory, Upton, United States, ${ }^{2}$ Photon Science. Brookhaven National Laboratory, Upton, NY, United States, ${ }^{3}$ Advanced Photon Source, Argonne National Laboratory, Lilse, Illinois, United States

E-mail: hanson1@bnl.gov

Copper doped ceria (CuCeria) has been shown to catalyze the water gas shift (WGS) reaction ( $\mathrm{H} 2 \mathrm{O}+\mathrm{CO} \rightarrow \mathrm{H} 2+\mathrm{CO} 2$ ). The $\mathrm{Cu}+2$ ion replaces $\mathrm{Ce}+4$ ion in the fluorite lattice at a shifted position that allows the $\mathrm{Cu}$ ion to have square planar oxygen coordination. The CuCeria has been shown to be active when the $\mathrm{Cu}$ atom leaves the fluorite lattice [1]. In order to better understand the transformation from CuCeria to $\mathrm{Cu}$ particles on ceria, we have made in situ measurements of CuCeria while cycling pulses of $\mathrm{O} 2$ and $\mathrm{CO}$. These measurements were made with a combined Diffuse reflectance Infrared Fourier Transform Spectroscopy (DRIFTS). XRD and PDF instrument at beamline 11ID-B at the Advanced Photon Source [2]. The changes in the series of PDF data (top Figure) during the transformation appear to be minor, but can be clearly seen when data from the cyclic series of PDF measurements are demodulated by the Phase Sensitive Detection (PSD) technique (bottom Figure) [3]. The data from the oxidized portion of the PSD (blue line) show the Cu-O of the Cu in the ceria lattice and the $\mathrm{Ce}-\mathrm{O}$ resolved from each other. The plot from the data in the reduced region (red line) shows the appearance of the shortest $\mathrm{Cu}-\mathrm{Cu}$ contact in the $\mathrm{Cu}$ metal formed on the surface of the ceria. The Ce-Ce distance peak in the oxidized region is $3.75 \AA$ and increases to $3.99 \AA$ in the reduced region. The "local" structure change in nearest Ce-Ce distance is much larger than the average structure value determined from the crystalline powder pattern; however is consistent with the expected short range changes. The PSD of the simultaneous DRIFTS measurement shows several spectral features that can be correlated with the PSD of the PDF changes. The advantage of PSD analysis of the time resolved (TR) series of PDF'S shows only changes to the amount of each PDF distance and the relative time that the change occurs. In this case, the changes during oxidizing and reducing conditions are resolved.

This work was supported by Brookhaven National Laboratory through a Brookhaven National Laboratory LDRD Project. Brookhaven National Laboratory is supported by the U.S. Department of Energy (DOE), Office of Science, Office of Basic Energy Sciences, under Contract No. DE-AC02-98CH10886. This research used resources of the Advanced Photon Source, a U.S. Department of Energy (DOE) Office of Science User Facility operated for the DOE Office of Science by Argonne National Laboratory under Contract No. DE-AC02-06CH11357.

1. Wang, X.Q., et al., (2006) JPCB, 110, 428-434

2. Beyer, K.A, et al,(2014) J. Appl. Cryst. 47, 95-101

3. Urakawa, A., et al., (2008) Chem. Eng. Sci. 63, $4902-4909$

TR PDF
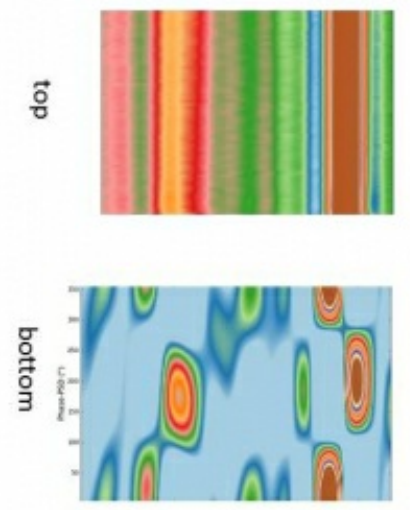

$\mathrm{r} / \mathrm{A}$
PDF of Reduced and oxidized CuCeria

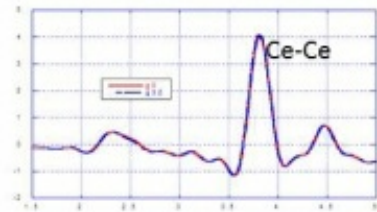

Raw PDF

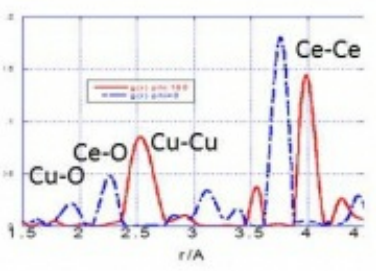

$r / \AA$

Keywords: In situ, PDF, Phase Sensitive Detection 